\title{
Tiled Projector Displays Correction for Dark Scenes in Railway Simulators
}

\author{
C. Zoido, J. Maroto, G. Romero, J. Felez \\ Research Group in Railway Technology and Advanced Simulation (CITEF) \\ Universidad Politécnica de Madrid, Spain \\ e-mail: czoido@etsii.upm.es, joaquin.maroto@upm.es, gregorio.romero@upm.es, jesus.felez@upm.es
}

\begin{abstract}
Tiled projector displays are a common choice for training simulators, where a high resolution output image is required. They are cheap for the resolution that they can reach and can be configured in many different ways. Nevertheless, such kinds of displays require geometric and color correction so that the composite image looks seamless. Display correction is an even bigger challenge when the projected images include dark scenes combined with brighter scenes. This is usually a problem for railway simulators when the train is positioned inside a tunnel and the black offset effect becomes noticeable. In this paper, a method for fast photometric and geometric correction of tiled display systems where dark and bright scenes are combined is presented. The image correction is carried out in two steps. First, geometric alignment and overlapping areas attenuation for brighter scenes is applied. Second, in the event of being inside a tunnel, the brightness of the scene is increased in certain areas using light sources in order to create the impression of darkness but minimizing the effect of the black offset.
\end{abstract}

Keywords-component; projection; simulator; black offset; blending

\section{INTRODUCTION}

Multi-Projector display systems are the most popular choice for $3 \mathrm{D}$ scene visualization in training simulation. Their capacity to achieve high resolutions over a wide fieldof-view with configurable aspect ratio makes them a very flexible solution. Recent advances in projection technology, 3D graphics hardware and capture devices make low cost calibration of such kinds of displays possible.

The aim in the design of tiled projector displays is to obtain a seamless output image. That means suppressing all perceptible discontinuities caused by geometric misalignment and color variations across the projectors while trying not to reduce the image quality. When the projection display is used for training, simulation time is crucial and a fast and completely automatic calibration is also a must.

In order for this goal to be reached there are several challenges and issues that must be addressed. First of all, casual projector placement has to be corrected making to ensure a perfect alignment [1]. This is usually achieved using a camera as input device to capture a known projected geometrical pattern. The analysis of these images makes it possible to establish transformations between each projector and the projection surface. After the geometric correction has been completed there are still noticeable photometric continuity breaks in overlapping areas and color shifts between projectors can also appear [2].
One of these photometric discontinuities is caused by the fact that projectors are unable to project "true black", instead they project a certain amount of light called black offset. This effect is negligible when images of bright and vivid colors are projected but it is very noticeable for dark scenes. Railway simulator display systems are affected by this issue as a significant part of the simulation takes place in low light environments, such as tunnels.

Previous work on black offset correction [3] usually tries to smooth black level variations across the display. This is done post processing the image to increase the minimum black level of the projectors. This leads to a loss of contrast typically producing a "washed out" image.

There are also commercial solutions based on neutral density filters in front of the lens that interfere the light path, reducing the black level in overlapping regions [4]. This method can achieve good results for dark only scenes like a whole simulation during night time but they cannot handle simulations with periodic transitions from bright to dark scenes.

The proposed implementation is specifically designed for simulators where dark environments are combined with brighter ones. This is a typical case in railway simulators where the train is constantly alternating dark and bright areas. The presented correction is divided into two steps. The first one corrects photometric discontinuities in bright scenes. The second one uses an additional correction in order to mitigate the effect of the "black offset" for dark scenes.

\section{TILED PROJECTOR DisPlays CORRECTION}

As previously stated, this kind of display needs to be corrected (in both geometry and color Fig. 1). Typical methods of correction are explained next.

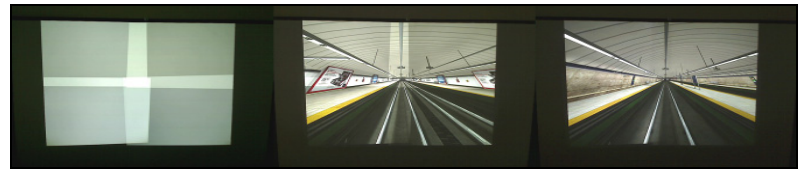

Figure 1. From left to right, initial projector positions, geometrically corrected display and display corrected in geometry and color.

\section{A. Geometric correction}

Using a camera to find out the geometric transformations between the camera itself, the projection surface and each projector's coordinate system is the most common approach. The coordinate systems for a planar surface are shown in Fig. 2 adopting the notation followed by [5]. 


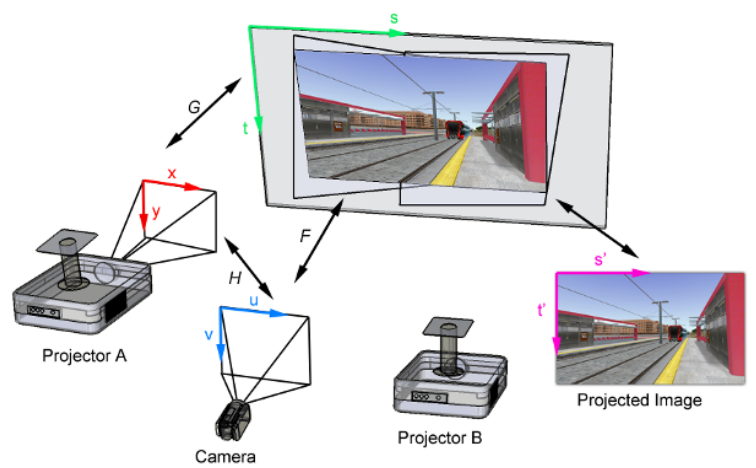

Figure 2. Coordinate systems involved in geometric correction for a planar surface.

The transformation that has to be found is given by

$$
G_{\left(x_{i}, y_{i}\right) \rightarrow(s, t)}=F_{(u, v) \rightarrow(s, t)} \cdot H_{\left(x_{i}, y_{i}\right) \rightarrow(u, v)} .
$$

Where $H$ is the transformation from the camera coordinates to the projector coordinates. $F$ is the transformation from the projection surface coordinates to the camera coordinates. Final displayed image coordinates $\left(\mathrm{s}^{\prime}, \mathrm{t}^{\prime}\right)$, and projection surface coordinates $(\mathrm{s}, \mathrm{t})$, are considered equivalent for planar surfaces as they are related by a scale plus a translation. $G$ is the sought relationship that transforms each projector to the projection surface.

There are several ways to find this transformation. If all the relationships between elements are considered linear [6], then $F$ and $H$ are homography mappings. If a linear assumption is not met, then the elements can be modeled with piecewise linear methods projecting a pattern with dense correspondences and using linear methods for interpolation between these features. Finally, non-linear models can be also used. Cubic polynomials [7] can be used to transform between camera and projector systems. Bezier patches [8] can also model the non-linearities present in these kinds of systems with great accuracy.

\section{B. Photometric correction}

Once the image has been geometrically corrected using any of the previous methods, shifts can still be perceived in the image due to the differences in brightness of the projectors.

There are different causes behind these differences in brightness:

- Variations within the projector's area of influence. These are usually mainly due to the vignetting effect which diminishes the projector's brightness at the edge of the images.

- Color variations between projectors. This normally occurs when different technology projectors are mixed together or are of the same technology but with very different usage times.
- Overlapping zones. In the overlapping zones between projectors the brightnesses are added to one another leading to a shift in the image.

Over the years different methods have been proposed to correct the color variations in this type of display. The influence of some of the above causes of variation is usually neglected in order to focus on correcting the major problems:

- Luminance attenuation maps. These only bear in mind the luminance throughout the projected image [9], correcting the variations in a single projector and between projectors in an attempt to produce smooth transitions between them to minimize the shifts in brightness.

- Gamut matching. These methods [10] usually use a color measurement device as a spectroradiometer to calculate each projector's color gamut. Once the gamut of each projector has been found, all are adjusted to one in common. This procedure is expensive and slow as a large number of measurements need to be made by radio spectrometry.

- Gamut morphing. Gamut morphing-based methods [11] attempt to produce smooth transitions between projectors by morphing the gamut between one projector and another instead of adjusting all to a common gamut as in the previous method.

In most of the corrections proposed previously the black offset effect is neglected or is corrected by post processing the image in each case in the same way that that the other causes of color variation are corrected. This way of correcting the effect does not give good results for very dark scenes, as will be seen in the next section.

\section{Proposed CORRECTION MODEL}

The purpose of the proposed correction is to minimize the negative effects caused by the black offset effect in dark scenes but without any loss of contrast in the image for bright scenes where this correction is no longer necessary. We are not going to deal with the color variations between projectors as it is usual for all the projectors to be identical and, in that case, color differences tend not to be great. Correction is a two-stage process. First, the shifts in brightness between projectors are corrected by applying attenuation masks to obtain smooth transitions in the overlapping zones. This correction would normally be sufficient for the bright scenes; however, since the bright scenes are continuously alternating with the dark scenes, a second additional correction is required to mitigate the black offset effect when representing dark scenes, losing the minimum sensation of lack of light when the image is seen.

\section{A. Correcting the overlapping zones for bright scenes}

The most obvious cause of image discontinuity for multiprojector systems where there is no major difference in the projectors' color range are the overlapping zones. The best way to ensure that the transitions between projectors through 
the overlapping zones are imperceptible is to apply masks to attenuate the brightness in those zones. These masks are generated according to the data obtained in the geometric correction process of the projectors. The same correction function $G\left(\vec{p}_{i}\right)$ is used as presented in [12] and is expressed as follows:

$$
G\left(\vec{p}_{i}\right)=\frac{\xi_{i}}{\sum_{j=0}^{N} \xi_{j}} ; \xi_{i}=\prod_{k=1}^{4} d_{i, k}
$$

Where $G\left(\vec{p}_{i}\right)$ is the attenuation factor by which each pixel is multiplied and $d_{i, k}$ is the distance from a point on the projector $i$ to the edge of each projector $k$ with which it overlaps.

It must be borne in mind that this correction cannot be applied directly because it is based only on the geometry of the projection system and takes no account of the projectors' transfer functions. These functions are not linear, and if the correction were to be applied directly an overcompensation effect would be produced like that shown in Fig. 3.

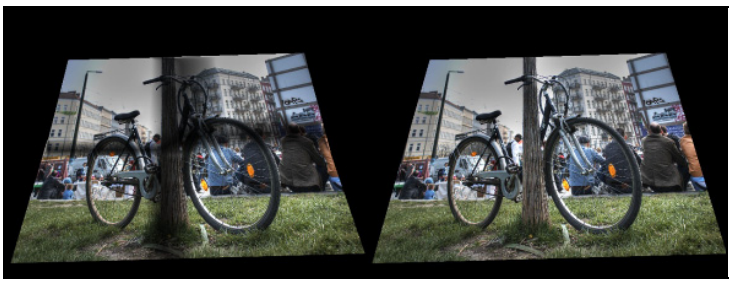

Figure 3. Correction mask applied directly (left) and with supplementary gamma correction (right)

To avoid this effect, the projector's transfer function is approximated to an exponential function of the exponent $\gamma$. Therefore, the final modified correction factor would be:

$$
A\left(\vec{p}_{i}\right)=G\left(\vec{p}_{i}\right)^{\gamma}
$$

This simplification furnishes a good result and avoids having to perform a prior calibration stage to estimate the projectors' transfer functions. By using this method a large amount of time can be saved compared to calibrating a new projector system.

This correction, in itself, would be sufficient to be able to see the images generated by the simulator, free of discontinuities for bright scenes. However, as can be seen from Fig. 4, this correction mask has no effect on dark scenes as it is impossible to lower the level of brightness of the black offset.

It is for this reason that a supplementary correction must be introduced. Instead of performing a post processing of the image, which will lead to no satisfactory solution for the scene types, this supplementary correction is performed by adding lights inside the graphic engine that generates the 3D graphics of the simulator.

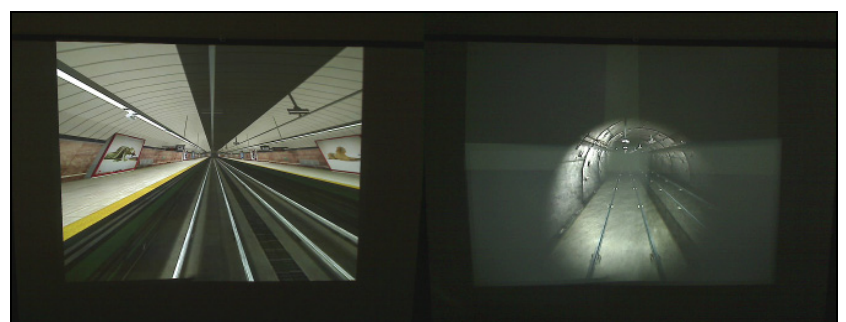

Figure 4. Correction only by mask for bright scenes in illuminated stations (left) and for scenes inside an unlit tunnel (right).

\section{B. Correcting the black offset effect in dark scenes}

When an image is constructed by means of the superposition of several projectors, there are overlapping zones. These overlapping zones, with the previous correction applied, are imperceptible when the brightness of the image in the zone or in its surroundings is high. However, when the color of the zone is close to black, the effect is highly noticeable.

In the case of underground railway simulators, for most of the time the train will be running through unlit tunnels. Therefore, in many cases, the only light emitted is from the train's headlights. Moreover, these lights are usually set to illuminate the track, leaving the roof of the tunnel in darkness. In this situation, the previously mentioned effect becomes particularly visible if the overlapping zone is centered on the projection surface.

In order to minimize the effect, the following alternatives have been studied:

1) To increase the brightness of the final projected image until the effect is hardly noticeable to the human eye: This consists of a displacement of the RGB to convert the pure black $\operatorname{RGB}(0,0,0)$ color into a color where the overlapping zone is not noticeable. The major problem with this method lies in the fact that the range of each of the color components is seen to be less. That is why, in illuminated environments the image appears to be less "alive" and in unlit environments, since the color black predominates, the resulting image will take on a greyish hue, with a reduction in the level of realism. The main advantage is that only the final image processing is affected with no requirement to modify the algorithm associated with the scene illumination calculation, and is therefore a method that can be easily applied to any simulator. So, the following transformation will be applied to each of the original color components:

$$
c^{\prime}=c \cdot\left(1.0-c_{b}\right)+c_{b}
$$

Where $c^{\prime}$ is the final color component after processing the image, $c$ is the color component before making the modification and $c_{b}$ is the applied displacement. 


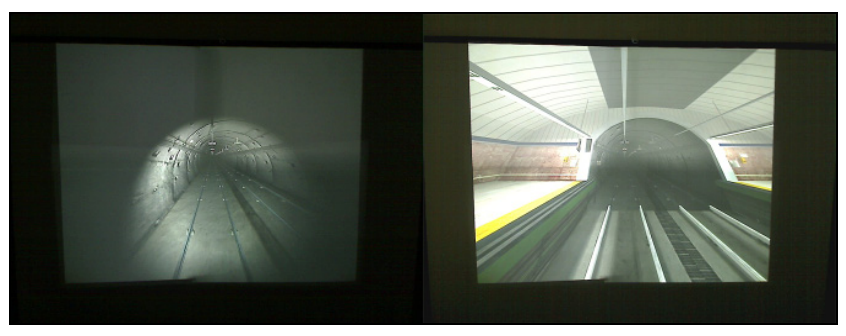

Figure 5. Correction by displacing and scaling the RGB curve associated with each projector.

2) To increase the global brightness of the unlit tunnel using ambient lighting: This consists in inserting ambient lighting $\left(l^{\prime}\right)$ that only affects the unlit tunnels. Its main advantage is the low computational cost. However, the drawback is that the unlit zones situated in the distance appear to be illuminated, as can be seen in Fig. 6, giving the impression of infinitely powerful headlights. In this case, the algorithm is applied as part of the illumination calculation; its computational cost, however, is very small.

$$
c^{\prime}=c+m_{D} \cdot l^{\prime}
$$

Where $c^{\prime}$ is the pixel color before applying the masks, $c$ the resulting color of the lighting model before applying $l^{\prime}$ and $m_{D}$ the diffuse component of the material.

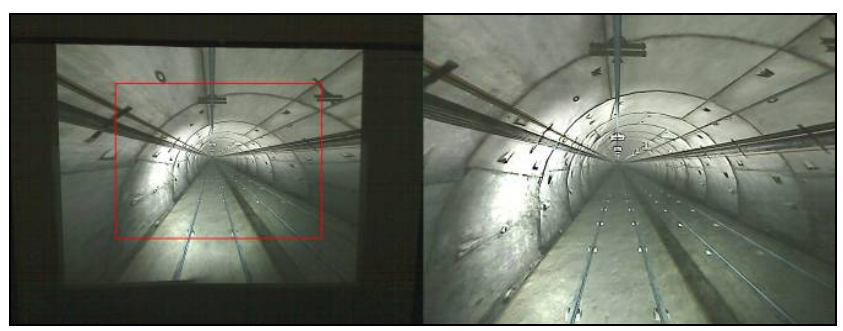

Figure 6. Correction by the insertion of ambient lighting.

3) To increase the global brightness of the unlit tunnel by displacing and scaling the color curve: This is similar to the first technique described but applied only to the unlit tunnels. Since the illuminated geometries are not affected by this algorithm the color displacement and scaling must only be applied within the lighting shader associated with unlit tunnels. The result would be similar to that shown in Fig .5 for the unlit tunnels.

$$
c^{\prime}=c \cdot\left(1.0-c_{b}\right)+c_{b}
$$

Where $c^{\prime}$ is the pixel color before applying the masks, $c$ the resulting color of the lighting model and $c_{b}$ the displacement of the graphics.
4) To increase the brightness of the zones closest to the viewpoint: This kind of illumination could be justified by a light emitted from the cab. Its main advantage is that it furnishes good sensations for both the zones close to the viewpoint, affected by the train's headlights, as well as for the distant zones that are unaffected by them. Its main drawback is the increase in computational load. However, at present, and thanks to shader-based lighting techniques [13] [14], its consumption is perfectly assumible. In this case, the component resulting from multiplying the diffuse color of the material by an attenuation function depending on the distance will be applied.

$$
c^{\prime}=c+\frac{m_{D}}{\left(k_{a}+k_{b} \cdot d+k_{c} \cdot d \cdot d\right)} .
$$

Where $d$ is the distance from the light source and $k_{a}, k_{b}$ and $k_{c}$ are the constant, linear and quadratic coefficients, respectively.

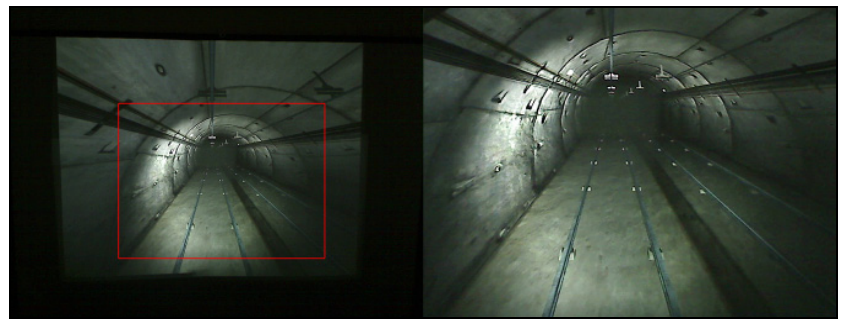

Figure 7. Correction by inserting an attenuated light according to distance.

The constant, linear and quadratic coefficients must be selected manually as they will depend on the residual brightness of the projectors, the color of the tunnel and the projection matrix, among other things.

This effect obviously illuminates all the zones close to the viewpoint up to a certain distance. However, the overlapping zone is usually located in the centre of the screen, a point where zones distant from the viewpoint are usually projected and therefore are not directly affected by the added light. However, the overlapping zone is not noticeable due to the fact that the illumination of the areas affected by the new light produces an increase in the average brightness of the overall image which indirectly causes the zone not to be noticed.

But the increase in brightness of the unlit tunnel Fig. 8a and the increase in brightness in the zones close to the viewpoint, Fig. $8 \mathrm{~b}$, produce an undesirable effect in the transitions between unlit tunnels and illuminated environments. In these circumstances, the case arises that the track inside the unlit tunnel is more illuminated than that in an illuminated station or exterior. 


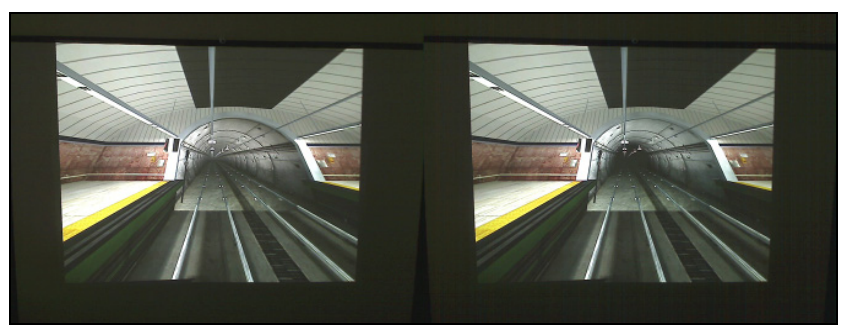

Figure 8. $\mathrm{a}$ and $\mathrm{b}$. Transitions between unlit tunnels and illuminated stations.

An initial solution would be to make the "imaginary light emitted by the cab" as well as the light emitted by the train's headlights to also affect the unlit stations or illuminated exteriors. However, this solution frequently produces a burning of the nearby zones, Fig. 9, as it is the result of the sum of the original light and the added light, leading to a drastic reduction in the level of realism.

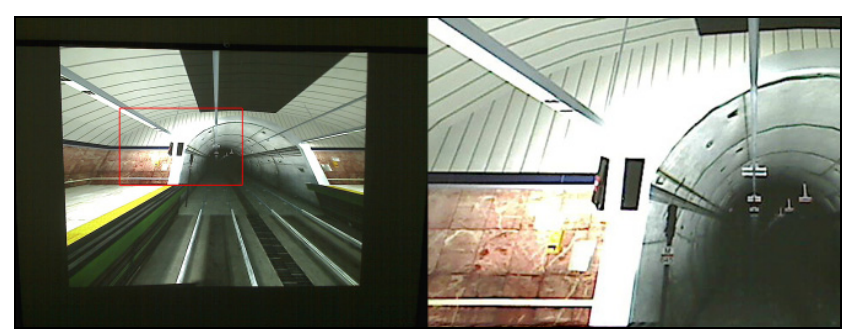

Figure 9. Burning of the image by light introduced through the minimization of the negative effects associated with the level of offset.

To avoid this effect a function needs to be introduced that is dependent on the distance of the closest illuminated environment, as well as its degree of illumination. By so doing, both the light from the train's headlights and the light emitted by the cab gradually diminish in intensity in the surroundings of the illuminated zones and disappear almost completely inside these zones.

This function attempts to simulate the human eye's adaptation to different levels of illumination. In an illuminated environment the light emitted by the train's headlights as well as the cab may not be noticeable. On the contrary, in unlit tunnels, since they are the only sources of light, the eye adapts to these lighting conditions and perfectly learns their effects.

This algorithm is not incompatible with the need to achieve a minimum brightness inside the overlapping zone, Fig. 10. In the surroundings of an illuminated environment, on the one hand, the effects of the light associated with the train will be reduced, but on the other, the illuminated stations and exteriors will increase their average brightness of the scene to a larger degree and so cause the level of offset to be less noticeable.

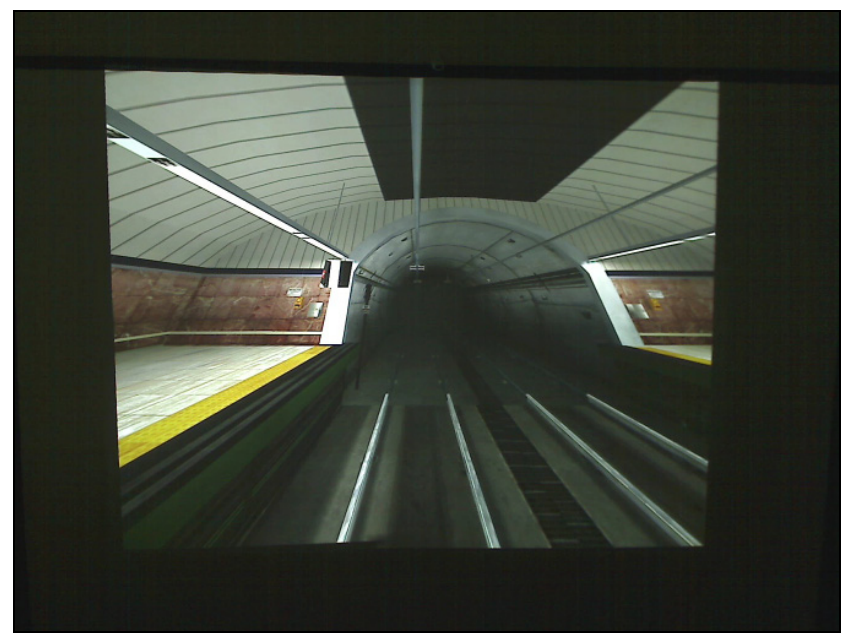

Figure 10. Resulting image taking account of the eye's ability to adapt to every lighting situation.

\section{SHADER IMPLEMENTATION}

The end result depends on two nested program shaders [15], [16]. The first is the one affecting the illumination of the scene and the second processes the ensuing image to apply the mask. Also included in the last program will be the correction described in (4), in the event of it being applied.

\section{A. Shader illumination}

Vertex shader:

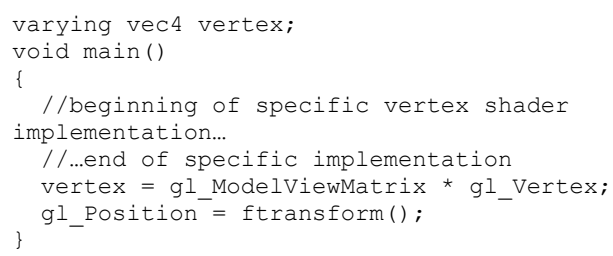

Fragment Shader:

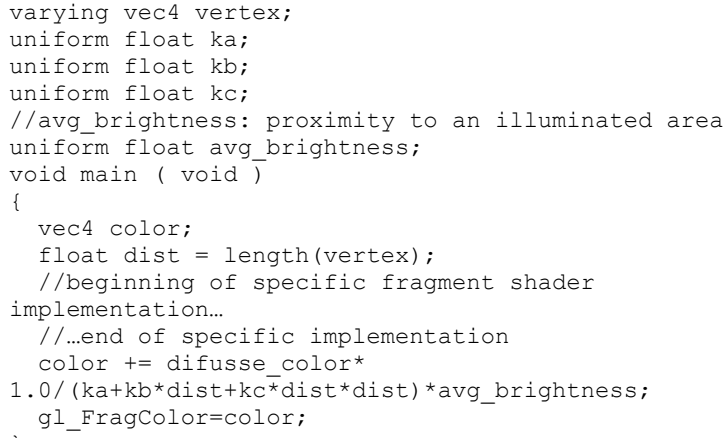




\section{B. Post-processing Shaders}

\section{Vertex shader:}

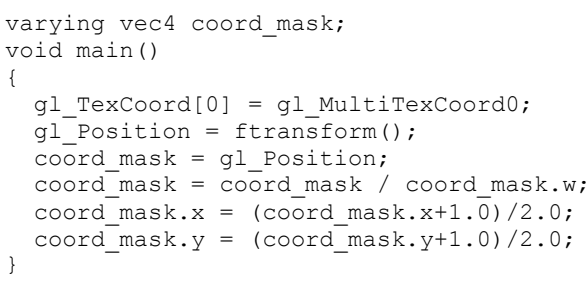

Fragment Shader:

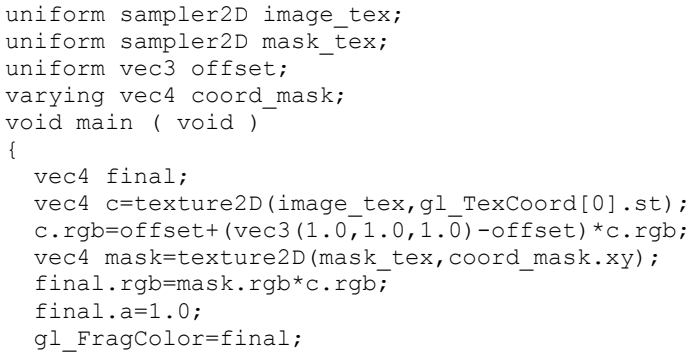

\section{CONCLUSIONS}

The proposed correction method has been applied to underground driver training simulator projection systems with good results. Correcting the image using the graphic engine instead of post processing the final rendered image enables the black offset to be minimized for dark scenes without degrading the image quality. The method is a general one and can be applied to any lighting model no matter how complex. The only limitation to the system is that it is only applicable to contents generated by a $3 \mathrm{D}$ graphic engine; that is, it would be of no use for projecting static images or video content.

\section{REFERENCES}

[1] A. Raij, G. Gill, A. Majumder, H. Towles and H. Fuchs, "PixelFlex2: a comprehensive, automatic, causally-aligned multiprojector display," PROCAMS'03.

[2] Niranjan Damera-Venkata, Nelson L. Chang and Jeffrey M. DiCarlo, " A Unified Paradigm for Scaleable Multi-Projector Displays, " IEEE Transactions on Visualization and Computer Graphics (Proceedings Visualization / Information Visualization 2007), vol.13, no. 6, pp. 1360-1367, Nov-Dec 2007.

[3] M. Brown, A. Majumder and R. Yang. 2005, "Camera-Based Calibration Techniques for Seamless Multiprojector Displays," IEEE Transactions on Visualization and Computer Graphics 11, 2 (March 2005), 193-206.

[4] A. Koebel, "Led Illumination for Simulation and Training," unpublished.

[5] A. Majumder and M. Brown, "Practical Multi-Projector Display Design,” A. K. Peters, Ltd., Natick, MA, USA. 2007.
[6] H. Chen, R. Sukthankar, and G. Wallace, "Scalable Alignment of Large-Format Multi-Projector Displays Using Camera Homography Trees," In Proceeding of IEEE Visualization 2002, pages 339-346, 2002.

[7] M. Hereld, I. R. Judson, and R. Stevens, "Dottytoto: A measurement engine for aligning multi-projector display systems," Argonne National Laboratory preprint ANL/MCS-P958-0502, 2002.

[8] Ezekiel S. Bhasker, Aditi Majumder, "Geometric Modeling and Calibration of Planar Multi-Projector Displays Using Rational Bezier Patches," Computer Vision and Pattern Recognition, IEEE Computer Society Conference on, pp. 1-8, 2007 IEEE Conference on Computer Vision and Pattern Recognition, 2007.

[9] A. Majumder and R. Stevens., "LAM: luminance attenuation map for photometric uniformity in projection based displays," In Proceedings of the ACM symposium on Virtual reality software and technology (VRST '02). ACM, New York, NY, USA, 147-154.

[10] G. Wallace, H. Chen, and K. Li, "Color gamut matching for tiled display walls," In Proceedings of the workshop on Virtual environments 2003 (EGVE '03). ACM, New York, NY, USA, 293302.

[11] B. Sajadi, M. Lazarov, M. Gopi, and A. Majumder, "Color Seamlessness in Multi-Projector Displays Using Constrained Gamut Morphing," IEEE Transactions on Visualization and Computer Graphics 15, 6 (November 2009), 1317-1326.

[12] Michael Harville, Bruce Culbertson, Irwin Sobel, Dan Gelb, Andrew Fitzhugh, and Donald Tanguay. 2006. Practical Methods for Geometric and Photometric Correction of Tiled Projector. In Proceedings of the 2006 Conference on Computer Vision and Pattern Recognition Workshop (CVPRW '06). IEEE Computer Society, Washington, DC, USA, 5-.

[13] Mike Bailey, Steve Cunningham, "Graphics Shaders: Theory and Practice", A. K. Peters, Ltd. Natick, MA, USA (C)2009,ISBN: 1568813341

[14] Mike Bailey, "Teaching OpenGL shaders: Hands-on, interactive, and immediate feedback", Computers \& Graphics, Volume 31, Issue 3, June 2007, Pages 524-531

[15] Randi J. Rost, "The Orange Book: The OpenGL Shading Language", July, 2009, Pages: 792, Addison-Wesley Professional, ISBN: 9780321637635

[16] Lambert M. Surhone, Mariam T. Tennoe, Susan F. Henssonow, "PerPixel Lighting”, Betascript Publishing, ISBN:9786133667556 